CIUDAD Y TERRITORIO

ESTUDIOS TERRITORIALES

ISSN(P): 1133-4762; ISSN(E): 2659-3254

Vol. LII, № 204, verano 2020

Págs. 307-320

https://doi.org/10.37230/CyTET.2020.204.08

CC BY-NC 4.0

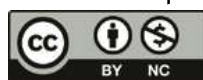

\title{
Áreas no municipalizadas y autonomía de los pueblos indígenas en Colombia
}

Naídú Duque-CANTE ${ }^{1}$

Profesora Investigadora Institución Universitaria Politécnico Grancolombiano. Bogotá, Colombia

RESUMEN: Las áreas no municipalizadas, presentes en los departamentos de Amazonas, Guainía y Vaupés en Colombia, son territorios que no coinciden con las entidades territoriales de nivel local definidas en la Constitución de 1991, por lo que la gestión de las mismas está en manos del departamento al que pertenecen. A partir de una revisión normativa y documental para identificar las razones y particularidades que han impedido su formalización como entidades territoriales se pudo determinar que estas áreas presentan características sociales, económicas y ambientales muy diferentes de los demás territorios de nuestro país. Los intentos por convertirlos en entidades territoriales o anexarlos a entidades de nivel local existentes han sido infructuosos debido a que los marcos normativos actuales no recogen las particularidades relativas a los vínculos entre el territorio y las comunidades indígenas que los habitan. El gobierno nacional ha optado por reconocerle cada vez más derechos a los Territorios Indígenas que las componen, ya que es muy poco probable que el congreso autorice la creación de las denominadas Entidades Territoriales Indígenas.

PALABRAS CLAVE: Áreas no municipalizadas; Territorios indígenas; Comunidades indígenas; Entidades territoriales; Departamentos.

\section{Non-municipalized areas and autonomy of indigenous communities in Colombia}

ABSTRACT: The non-municipalized areas, present in the departments of Amazonas, Guainía and Vaupés in Colombia, are territories that do not coincide with the territorial entities of local level defined in the 1991 Constitution, so that their management is in hands of the administration of the department to which they

Recibido: 18.01.2019; Revisado: 28.03.2019

Correo electrónico: naduquec@poligran.edu.co naidu.duque@gmail.com NºRCID: https://orcid.org/0000-0003-2129-7272 La autora agradece los comentarios y sugerencias realizados por los evaluadores anónimos, que han contribuido a mejorar y enriquecer el manuscrito original.

${ }^{1}$ Este artículo es uno de los productos del Proyecto de Investigación "Categorización Municipal en Colombia" el cual se desarrolla en el marco del Programa de Doctorado en Modelado en Política y Gestión Pública de la Universidad Jorge Tadeo Lozano que cursa actualmente la autora. El Proyecto es financiado en su totalidad por la Institución Universitaria Politécnico Grancolombiano (IUPG), Bogotá (Colombia) y pertenece a la línea de Administración Pública del grupo de investigación Economía, Derechos y Globalización de la IUPG. 
belong. From a normative and documentary review to identify the reasons and particularities that have prevented its formalization as territorial entities, it was determined that these areas have social, economic and environmental characteristics very different from the other territories of our country. Attempts to convert them into territorial entities or annex them to existing local level entities have been unsuccessful because the current regulatory frameworks. They do not reflect the particularities related to the links between the territory and the indigenous communities that inhabit them. The national government has chosen to recognize more and more rights to the Indigenous Territories present in these areas, because it is very unlikely that the Congress will authorize the creation of the so-called Indigenous Territorial Entities.

KEYWORDS: Non-municipalized areas; Indigenous territories; Indigenous communities; Territorial entities.

\section{Introducción}

T. diseño territorial es la base a partir de la cual se establecen los criterios de gestión de los asuntos que tienen lugar al interior de un país. Pero tanto el diseño del territorio como la gestión que se haga del mismo constituyen los medios a través de los cuales se busca mejorar los niveles de bienestar social y económico de la población. Por este motivo, es trascendental que cada país, atendiendo a sus ámbitos y necesidades propios decida cuál es la fórmula territorial que más le conviene. Colombia, como producto de la maduración institucional de que ha sido objeto, cuenta actualmente con dos tipos de entidades territoriales de nivel local y una de nivel intermedio. Sin embargo, y a pesar de la evolución de sus marcos normativos, todavía no ha logrado incorporar la totalidad de su territorio dentro de estas categorías, lo cual ha derivado en la existencia de áreas no municipalizadas.

Una revisión de la información aportada tanto por el diseño normativo existente como por las fuentes documentales referidas a la temática permitieron comprender de manera más clara cuales han sido las circunstancias que han impedido la formalización de estos territorios. Para ilustrarlo, este documento se encuentra estructurado en cinco partes centrales que incluyen el marco teórico y conceptual, seguido de la descripción del diseño territorial colombiano con el fin de comprender la ubicación de las áreas no municipalizadas en su interior. En tercer lugar, se analizan los intentos que se han realizado desde el diseño normativo para convertir a estas áreas en entidades territoriales, y las razones por las cuales no se logró este propósito. En cuarto lugar, se revisan las particularidades económicas, sociales y geográficas de estas zonas, seguida de la revisión de las normas que han sido recientemente expedidas para reglamentar la administración de estos territorios, a partir de los Territorios Indígenas. Finalmente, se realiza una revisión sobre el estado de avance a nivel latinoamericano en materia de creación de entidades propias para los pueblos indígenas.

\section{Marco teórico y conceptual}

El estado moderno le ha otorgado una importancia especial al territorio, en tanto el mismo representa el ámbito fundamental dentro del cual tienen sentido los demás elementos de su existencia. La necesidad de garantizar la defensa de la soberanía, el ejercicio del poder y el desarrollo de las dinámicas sociales, económicas y ambientales han conducido a la adopción de diferentes mecanismos de protección del territorio, así como formas de organización y gestión interna del mismo (GuARDERAS, 2007; LLANOS, 2010). En el proceso, los estados han incorporado alternativas de diseño espacial particulares, y que, en el caso de Colombia, han resultado de procesos de evolución que consultaron dinámicas sociales y económicas propias y heredadas de la época colonial.

La división político-administrativa que nos identifica en la actualidad, por ejemplo, ha sido fuertemente influenciada por el diseño territorial francés tanto previo como posterior a la Revolución el cual fue introducido a nuestro país a través de la corona española (HERNÁNDEZ, 2015). El centralismo histórico que nos ha caracterizado en estos dos siglos, así como la presencia de diseños normativos homogéneos para cada tipo de entidad territorial son muestra de este fenómeno. El derecho a la igualdad, introducido en Francia para regular las relaciones entre los individuos, también fue tomado en cuenta a la hora de realizar el reparto y manejo de los territorios (HERNÁNDEZ, 2018). Debido a esto, las normas que rigen a nivel subnacional no consultan las diferencias ambientales, sociales o económicas existentes entre entidades que pertenecen a la misma especie, derivando con ello en la presencia de dos tipos de fenómenos que no necesariamente están articulados. Por un lado, está la representación jurídica de la entidad territorial y, por otro, el fenómeno real compuesto por manifestaciones de tipo socioeconómico de carácter diverso.

Esta particularidad se puede observar en el caso de los municipios, los departamentos y los distritos, 
con la excepción de Bogotá, que cuenta con un régimen especial de administración y gestión (Decreto 1421 de 1993). Y aunque las normas relativas a la categorización territorial (Ley 136 de 19914, Ley 617 de 2000 y Ley 1551 de 2012) no desarrollaron el cometido establecido por la Constitución Política de 1991, que buscaba que desde el diseño institucional se reconocieran las diferencias existentes al interior de cada uno de los niveles del Estado (Artículo 320), esto no ha afectado la continuidad del Estado ni ha frenado la gestión de los territorios.

No obstante, los límites mínimos en el diseño institucional existente, de todas maneras, no han logrado cubrir la globalidad de las condiciones presentes en las regiones, dando lugar a la existencia de lo que se conoce como áreas no municipalizadas.

Su existencia evidencia la inoperancia del modelo de homogeneización territorial frente a realidades que cuentan con fenómenos tan particulares que no logran ser incluidos en los diseños tradicionales, en este caso para el nivel local. La consideración de estos fenómenos, entonces, debería partir de interpretar el territorio más allá de la división político-administrativa, para incorporar una comprensión del mismo como un "espacio apropiado por un grupo social para asegurar su reproducción y la satisfacción de sus necesidades vitales, que pueden ser materiales o simbólicas" (GIMÉNEZ, 2005, pp. 3). Esta apropiación, para el caso de las áreas no municipalizadas en Colombia, puede tener un carácter de mayor predominancia simbólico-cultural que utilitaria y funcional (RAFFEstin, 1980).

Esto se debe principalmente a que estas áreas han sido habitadas tradicionalmente por pueblos indígenas cuyo arraigo con el territorio ha sido reconocido de manera distinta al de las demás poblaciones. En ese sentido, además de considerar al territorio como un medio de subsistencia y fuente de recursos importantes, se le tiende a valorar primordialmente como el lugar donde habitaron sus antepasados y donde se encuentra anclada su historia y sus tradiciones (UNICEF, 2003). Él mismo, adquiere características sagradas y es fuente de la identidad que define a cada grupo humano que lo habita y sobre el cual ejerce influencia (BARABÁs, 2003).

En los territorios indígenas predomina la concepción del territorio como elemento simbólico y metafísico, el cual ha sido moldeado e interpretado por las comunidades que lo han ocupado tradicionalmente a partir de dispositivos inmateriales propios (UNICEF, 2003). En este proceso, el territorio es en sí mismo un ámbito donde hacen presencia las instituciones, las creencias y las prácticas culturales propias que han sido construidas e influidas por éste a través del tiempo condicionando la emocionalidad y el apego de los individuos y por ende la construcción de identidades y de cohesión social (FLORES, 2007). De igual manera, las manifestaciones culturales de estos pueblos pueden haberse encarnado directamente en el paisaje mediante lo que DEMARCHI (1983) describe como la dimensión ecológica de la cultura regional, expresada en paisajes rurales y urbanos, los monumentos, los caminos, canales de riego o cualquier otro tipo de antropoización de la naturaleza.

Esta interpretación del territorio, que debería estar en la base de la concepción de la entidad territorial, es distinta a la que se encuentra descrita en la Constitución Política de 1991 para el nivel local, donde se concibe al municipio como la entidad fundamental de la división político-administrativa del estado, la cual es responsable de prestar servicios públicos, construir obras, ordenar el desarrollo territorial, entre otras (Articulo 311). Aunque la concepción del nivel local podría haber partido de la consideración de los aspectos culturales, sociales e históricos característicos de las comunidades presentes en su interior, la carta magna prefirió continuar con la visión administrativista y legalista que describe PÉREZ (2010), en la que el municipio es apenas una entidad pública responsable de cumplir funciones y administrar recursos.

Por este motivo, la existencia de territorios locales no formalizados es un reto para las concepciones tradicionales sobre el territorio, en especial porque los territorios indígenas no se pueden equiparar a los demás municipios en Colombia. Esta particularidad ha sido incluida ya en Normas internacionales sobre la materia, como el Convenio № 169 de la Organización Internacional del Trabajo (OIT) (1989) y en la Declaración de las Naciones Unidas sobre los Derechos de los Pueblos Indígenas (2007). A través de estas normas se ordena a los estados, entre otros aspectos, el reconocimiento de derechos de los pueblos indígenas en materia de ocupación, posesión y participación de los beneficios derivados del territorio. Esto tomando en cuenta los vínculos ancestrales de estas poblaciones con el espacio que han ocupado y con el significado simbólico, sagrado y cultural que éste representa. En el proceso, además, los estados deberían reconocerles derechos políticos y la posibilidad de autodeterminación y manejo de sus asuntos propios a través de la creación de entes autónomos.

\section{Las áreas no municipalizadas en el diseño territorial en Colombia}

Colombia se compone actualmente, además del nivel nacional del Estado, de dos niveles 
territoriales claramente identificables en los que se incluyen los municipios y los distritos para el nivel local y los departamentos en el nivel intermedio. Esta configuración territorial, que se describe en el artículo 286 de la Constitución Política (CP) de 1991, es la condensación de cambios y transformaciones que tienen su origen desde la misma conformación de la República en el siglo XIX. A partir de ese momento el nivel local ha sido objeto de leves pero importantes modificaciones, en tanto el origen de la República derivó en la aparición de las parroquias (CP 1819), las cuales evolucionaron en los distritos parroquiales (CP 1832), que dieron paso a los distritos municipales (CP 1863), los que, a su vez, se convirtieron para el siglo $X X$ en dos tipos distintos de entidad territorial, los municipios y los distritos (Acto legislativo 01 de 1936) los cuales han trascendido hasta hoy.

Además de estas dos entidades, que constituyen una tradición en nuestro contexto, la Constitución de 1991 incluyó a las Entidades Territoriales Indigenas (ETIs) como posibilidades para el nivel local. Estas últimas fueron consideradas con el ánimo de contribuir al reconocimiento y consolidación de las autonomías de los pueblos indígenas que habitan nuestro territorio. Dicha posibilidad, aunque evidencia el ánimo garantista de nuestro estado, sigue vigente, ya que la Ley Orgánica de Ordenamiento Territorial (ley 1454 de 2011), que de acuerdo con la Constitución de 1991 debía ser la norma que institucionalizara su existencia, no las incluyó como entidades territoriales. Esto condujo a que los pueblos indígenas de nuestro país hayan tenido que continuar sujetándose a las directrices y procedimientos que emanan de las autoridades de los municipios o departamentos a los cuales pertenecen sus territorios, en asuntos que de otra manera podrían manejarse directamente por sus autoridades y responder a sus lógicas particulares (BAENA, 2015).

El nivel intermedio, por su parte, ha sido objeto de múltiples transformaciones ya que, aunque Colombia nació como estado unitario, lo hizo en un momento en el que el modelo federal también formaba parte del contexto político imperante (SIXIERI, 2014). Esta dualidad derivó en que, en la segunda mitad del siglo XIX, nuestro país se convirtiera en un estado federal, pero en un ambiente que se había caracterizado por un manejo altamente centralizado de los asuntos públicos dentro del modelo unitario. El resultado fue el fracaso de la propuesta federal y el consecuente retorno al estado unitario a finales del siglo XIX (1886) cuya configuración inicial se mantendría casi inalterada durante la mayor parte del siglo XX con la conocida formula "centralización política y descentralización administrativa". No obstante, la consolidación plena de la descentralización territorial, tal como la conocemos en la actualidad, se logró solamente a través de la elección popular de alcaldes (HERNÁNDEZ, 1999), introducida como reforma constitucional en el año 1986 (Acto legislativo 01) y que fue recogida luego mediante la Constitución política de 1991.

Todo este proceso tuvo implicaciones importantes, en especial para el nivel intermedio, que en el origen (CP 1821) estuvo compuesto por tres tipos de entidades distintas, los cantones, las provincias y los departamentos. Estas entidades respondían a tamaños geográficos diferentes, y a lógicas que no coinciden con las que rigen el diseño territorial en la actualidad. Lo anterior debido a que, en el caso particular de los departamentos, por corresponder en tamaño a cada uno de los tres países que formaban parte de la Gran Colombia, no tenía ninguna relación con los departamentos tal como los conocemos hoy. Sin embargo, estas entidades no tuvieron una larga existencia ya que, con la disolución de este estado, nuestro país conservó solamente las provincias y los cantones, pero solo fue hasta la Constitución de 1853 cuando también prescindió de estos últimos quedándose con una sola entidad de nivel intermedio. Las provincias tampoco tuvieron una larga duración, ya que al transitar hacia el modelo federal (Constituciones de 1858 y 1863) cambiaron su denominación a estados con la posibilidad de definir sus propios marcos constitucionales y legales.

Con el regreso al modelo unitario, por las razones económicas, sociales y políticas que llevaron a la expedición de la Constitución de 1886, los denominados estados pasaron a llamarse en adelante departamentos, los cuales, además, podían subdividirse para crear más de estas entidades como sucedió a lo largo del siglo XX, llegando a los 32 con que contamos en este momento. No obstante, para esta época ya existían ciertos territorios que, aunque solo fueron reconocidos en la Carta constitucional hasta el año de 1945 como entidades territoriales, formaban parte de nuestra realidad territorial y se conocían como Territorios Nacionales (GómEZ, 2010). Con la reforma del año 45 (acto legislativo 01) y a lo largo del siglo pasado estas entidades se formalizaron como Intendencias y Comisarías, y si bien eran entes intermedios y correspondían al mismo nivel geográfico de los departamentos, sus particularidades económicas, ambientales y geográficas los ubicaron por debajo de éstos, por lo que su administración se realizaba, principalmente, desde el nivel nacional (GómEZ, 2010).

Con la Carta constitucional del año 91 las intendencias (San Andrés, Aruca, Casanare y Putumayo) y comisarías (Vichada, Guaviare, Guainía, Vaupés y Amazonas) que todavía existían pasaron 
a llamarse departamentos (artículo 309), con lo cual se unificó la denominación de los entes de nivel intermedio. Además, se incluyeron las regiones (artículos 286, 306 y 307) y nuevamente las provincias (artículos 286 y 321 ) como alternativas para nuestro ordenamiento territorial. Aunque lo anterior podría haber derivado en la existencia de tres tipos de entidades en este nivel del Estado con tamaños geográficos y denominaciones distintas, en tanto las provincias se compondrían de municipios y entes indígenas, los departamentos de provincias, y las regiones de departamentos, la Ley Orgánica de Ordenamiento Territorial (LOOT) no autorizó tampoco su creación igual como sucedió con las ETIs. Por lo tanto, los departamentos continuaron siendo las únicas entidades territoriales de nivel intermedio y los municipios y distritos las del nivel local.

A pesar de la evolución constante que ha sufrido nuestro diseño territorial, el país se encuentra dividido en departamentos, pero no todo el territorio está dividido en municipios y distritos en tanto algunas áreas geográficas no encajan en estas alternativas. Estos territorios recibieron inicialmente la denominación de "corregimientos departamentales", y actualmente se les conoce como "áreas no municipalizadas" de acuerdo con algunas normas que reconocen su existencia (Decreto 2274 de 1991 y la ley 1551 de 2012). Se trata de entidades que no responden a una lógica constitucionalmente establecida, porque no alcanzan a cumplir con los requisitos de población y recursos para convertirse en municipios (artículo 11 de la ley 1551 de 2012). Los mismos son el rezago de los antiguos corregimientos intendenciales y comisariales que fueron reconocidos anteriormente mediante la ley 2 de 1943 y reglamentados con el Decreto 2451 de 1943. Dichas normas mencionan los setenta y dos (72) corregimientos en que se dividían las entonces intendencias del Chocó, Meta, y San Andrés y Providencia, y las comisarías de Arauca, Amazonas, Caquetá, Guajira, Putumayo, Vaupés y Vichada.

Debido a que las áreas no municipalizadas son una realidad que solo ha estado presente en los antiguos territorios nacionales, su existencia pone en evidencia defectos que van más allá de la presencia de vacíos en nuestro diseño normativo. La circunstancia de que estos territorios sean característicos de entidades que antes eran consideradas de categoría inferior a los demás departamentos y que con la Constitución ascendieron de estatus muestra que los cambios de que fueron objeto son de forma, pero no de fondo. Las características económicas, sociales y ambientales que marcaron las diferencias en el pasado siguen presentes en la dinámica de los nuevos departamentos, los cuales se componen en un alto porcentaje de poblaciones indígenas y están integrados por territorios principalmente selváticos que, por ser de difícil acceso, no permiten el desarrollo de la economía en las mismas condiciones y con características similares a las del resto de los departamentos en nuestro país (GoNZÁLEZ, 2010).

\section{Intentos de conversión de las áreas no municipalizadas en entidades territoriales}

Luego de la transformación de las últimas Intendencias y Comisarías en departamentos en 1991 (Ver FIG. 1), ha habido tres posibilidades para la conversión de los antes denominados corregimientos departamentales en entidades territoriales o en parte de entidades ya existentes. La primera de ellas fue el Decreto 2274 de 1991 el cual estableció que las áreas que no formaran parte de un municipio, y que todavía existían como corregimientos de las antiguas intendencias y comisarías debían mantenerse como divisiones departamentales (Artículo 21). El manejo de las mismas quedaría en manos de un Corregidor, agente del Gobernador y una Junta Administradora. Sin embargo, para el año 2001, la Corte Constitucional declaró inexequible esta disposición, y le dio al Congreso un plazo máximo de dos legislaturas para expedir un régimen que permitiera la transformación de los corregimientos departamentales en municipios o su incorporación al interior de municipios ya existentes (Sentencia C-141-01).

Pero, dado que han transcurrido 17 años sin que se haya cumplido esta ordenación, estos territorios ahora se encuentran en un limbo jurídico, ya que por un lado no están formalizados normativamente y por otro, su denominación se mantendría vigente, de acuerdo con la Corte, durante el periodo de transición correspondiente a las dos legislaturas que tenía el Congreso para su formalización. Ante la evidente inconstitucionalidad de la expresión "corregimientos departamentales" las normas más recientes que se refieren a ellos lo hacen como "áreas no municipalizadas", denominación que tampoco se encuentra definida desde la Constitución de 1991.

Pero este no ha sido el único intento por incorporar estas áreas dentro de la institucionalidad territorial. Otro de los casos más claros fue el artículo 44 de la ley 1551 de 2012, también declarado inexequible por la Corte Constitucional (Sentencia C-100-2013), el cual buscaba convertir los 21 corregimientos departamentales existentes (Ver FIG 2), en "territorios especiales biodiversos y fronterizos". La inexequibilidad se basó no solo en 
que se le estaba asignando una denominación especial a estas zonas, sino, principalmente, porque se buscaba mediante una ley y no la Constitución, reconocerlos como entidades territoriales al atribuirles derechos propios de éstas, como la posibilidad de elegir popularmente a sus autoridades político-administrativa

Adicionalmente, antes de esta ley, el legislativo tuvo la posibilidad, de formalizar las denominadas ETIs mediante la LOOT. Estas entidades, que sí están consideradas como alternativa desde la Constitución Política de 1991 (artículo 329), constituían una opción viable para garantizar el manejo de los asuntos locales de manera directa por parte de las poblaciones allí asentadas. Se trataría de entidades autónomas integradas principalmente por comunidades indígenas, con autoridades propias, y con funciones y recursos fiscales para la administración de sus propios intereses (Artículos 329 y 330 de la CP de 1991). Lo anterior podría haber conducido a la conversión en entidad territorial de los resguardos indígenas que se encuentran al interior de algunos municipios, pero principalmente habría permitido la formalización progresiva de las áreas no municipalizadas.

Debido a la falta de reconocimiento de estos territorios como entes autónomos de nivel local, su administración y el manejo de sus asuntos particulares ha estado en manos de los departamentos de los que forman parte. En consecuencia, estos departamentos han tenido que asumir responsabilidades que corresponden a dos niveles del Estado, el nivel intermedio y el nivel local. Esto se debe, en el segundo caso, a que, aunque la responsabilidad de garantizar un importante número de bienes y servicios públicos debería estar en manos de autoridades locales, su ausencia no

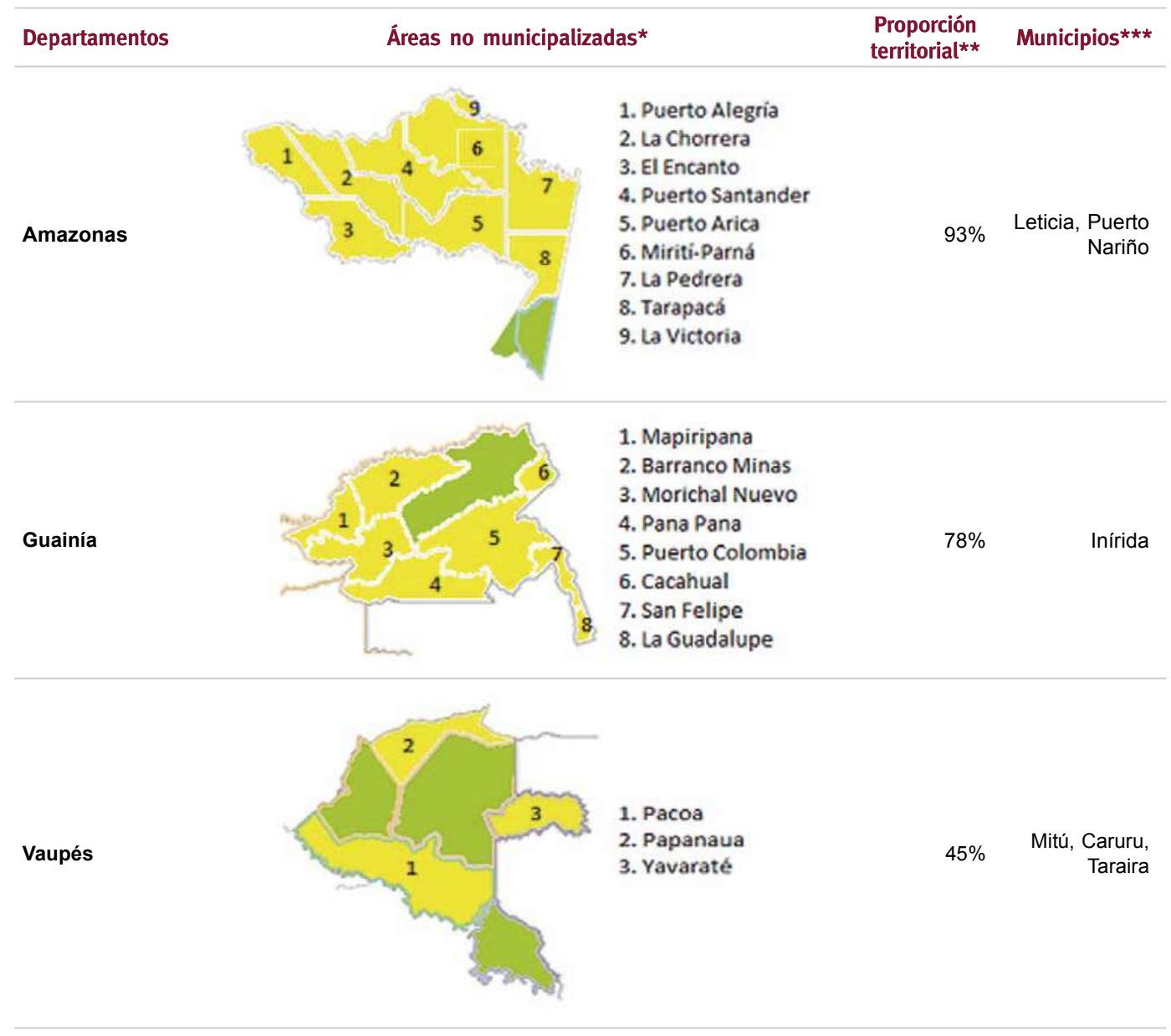

FIG. 1/ Áreas no municipalizadas en Colombia.

Fuente: Elaboración propia con base en la Ley 1551 de $2012^{\star}$, HURTADO, 2010* y Contaduría General de la Nación, $2018^{\star \star \star}$.. 
exime de responsabilidad al Estado. Incluso, para estas entidades el cumplimiento de las funciones de carácter local ha dejado de ser un tema residual, ya que las áreas no municipalizadas ocupan una gran parte de su territorio, siendo el caso más significativo el departamento del Amazonas donde el $93 \%$ de su área geográfica se distribuye en 9 de estos territorios, seguido del Guainía con el $78 \%$ de su territorio organizado en 8 de ellos y Vaupés con el $45 \%$ del suyo dividido en tres (Ver FIG. 1).

Además, dada la gran complejidad que se deriva de las funciones que forman parte de las administraciones locales en Colombia y las particularidades de tipo económico, social y geográfico de estas zonas, las autoridades departamentales deben enfrentar retos importantes con los que los departamentos tradicionales no tienen que tratar. Aunque su carácter selvático ha conducido a que en los últimos años se les preste mayor atención por su importancia ambiental, actualmente enfrentan problemas de deforestación, contaminación, minería ilegal y los efectos del cambio climático (CEPAL, 2014). La mayoría de sus habitantes pertenecen a comunidades indígenas, pero están sometidas a gobiernos no indígenas, bajo condiciones y reglas que fueron establecidas para los demás territorios. $\mathrm{Y}$, por último, las características geográficas no han permitido que se desarrolle una economía como en el resto del país, por lo que históricamente no han sido prioritarios para el gobierno central (GonZÁLEZ, 2010).

\section{Características económicas, sociales y geográficas de las áreas no municipalizadas en Colombia}

Las áreas no municipalizadas y principalmente los departamentos en los que se encuentran normalmente se ubican en los lugares más bajos en las descripciones y clasificaciones que se realizan tanto desde las fuentes oficiales como académicas en lo que respecta a los ámbitos sociales y económicos (Ver FIG. 2). Aunque estos territorios se caracterizan por contar con amplias zonas de bosques, humedales y zonas de reserva ambiental que superan en tamaño a todos los demás departamentos de Colombia (DNP 2017), también se identifican por tener niveles de densidad poblacional que están muy por debajo del promedio nacional y que ni siquiera alcanzan 1 habitante por $\mathrm{Km}^{2}$

${ }^{2}$ Las fuentes oficiales que ofrecen información sobre el nivel local en Colombia no incluyen a las áreas no municipalizadas, por lo que las referencias que se tomaron en cuenta en esta
Pero las diferencias más sobresalientes se presentan en los ámbitos social y económico, dado que se trata de entes que concentran las mayores proporciones de poblaciones indígenas del territorio nacional. Esta característica, es propia de los departamentos ${ }^{2}$ de Vaupés, Guainía, Guajira, Vichada, Amazonas, Cauca y Putumayo, entre los que se destacan Vaupés y Guainía con poblaciones indígenas por encima del $60 \%$ del total de habitantes. Estos departamentos también están entre los territorios con el mayor Indice de Pobreza Multidimensional (IPM) (CARMONA, SUPELANO $\&$ al. OSEJO (2014)).

Lo anterior coincide, además, con bajos niveles de crecimiento económico, ya que los departamentos que menos aportaron al PIB durante el año 2017 fueron en orden descendente La Guajira, Quindío, Sucre, Caquetá, Arauca, Chocó, Putumayo, San Andrés y Providencia, Amazonas, Vichada, Guaviare, Guainía y Vaupés. La mayoría de estas entidades se han caracterizado históricamente por una economía predominantemente agrícola y ganadera y con un auge reciente de la minería (DANE, 2017). En consonancia con lo anterior, el sector público de estas entidades registra los niveles más bajos de recaudo tributario, como se desprende del informe del Departamento Nacional de Planeación (DNP) del año 2017 sobre desempeño fiscal de los departamentos y municipios, en el que los peor ubicados fueron Guaviare, Vichada, Guainía, Amazonas y Vaupés. Estos últimos además son los departamentos que mayores niveles de transferencias por habitante reciben en el país, y en los que el nivel de dependencia de las transferencias supera el $70 \%$ del total de sus ingresos. Con lo cual se trataría de entes que funcionan más como unidades desconcentradas del nivel nacional que como entidades descentralizadas con autonomía territorial.

Otro punto de referencia sobre estos territorios se puede encontrar en algunos estudios orientados a realizar clasificaciones o categorizaciones de las entidades territoriales de acuerdo con las similitudes existentes en los ámbitos social, económico y ambiental (FIG. 3). Muchos de estos análisis, que normalmente son de carácter académico no oficial, han realizado clasificaciones tanto de los departamentos como de los municipios y algunos de ellos han incluido también las áreas no municipalizadas como parte del nivel local. Este es el caso de Ramírez \& Aguas (2016), Carriazo \& al. (2012) y PosAdA (2010), cuyos análisis parten de información como la cantidad de población que

parte aluden a los departamentos dentro de las cuales se incluyen estos territorios. 


\begin{tabular}{|c|c|c|c|c|c|c|c|c|}
\hline \multirow[b]{2}{*}{ DEPARTAMENTOS } & \multicolumn{2}{|c|}{ POBLACIÓN } & \multirow{2}{*}{$\begin{array}{c}\text { IPM } \\
\text { Índice } \\
\text { de pobreza } \\
\text { multidi- } \\
\text { mensional }\end{array}$} & \multicolumn{2}{|c|}{ ECONOMÍA } & \multicolumn{3}{|c|}{ SERVICIOS PUBLICOS } \\
\hline & $\begin{array}{c}\text { Indigenas } \\
\text { (\%) }\end{array}$ & $\begin{array}{l}\text { Densidad } \\
\text { Población }\end{array}$ & & $\begin{array}{c}\text { Partici- } \\
\text { pación } \\
\text { en el } \\
\text { PIB }\end{array}$ & $\begin{array}{l}\text { Ingresos } \\
\text { públicos } \\
\text { (Miles de } \\
\text { Pesos) }\end{array}$ & $\begin{array}{l}\text { Cobertura } \\
\text { de acue- } \\
\text { ducto (\%) }\end{array}$ & $\begin{array}{l}\text { Cobertura } \\
\text { neta en } \\
\text { educación } \\
\text { primaria }\end{array}$ & $\begin{array}{l}\text { Cobertura } \\
\text { neta en } \\
\text { educación } \\
\text { secundaria }\end{array}$ \\
\hline Amazonas & 43,4 & 0,69 & 0,74 & 0,1 & 22.039.322 & 61,41 & 80,27 & 59,77 \\
\hline Arauca & 2,2 & 10,89 & 0,59 & 0,5 & 34.275 .751 & 73,60 & 73,41 & 56,60 \\
\hline Caquetá & 1,6 & 5,30 & 0,71 & 0,5 & 29.520 .498 & 56,93 & 74,90 & 54,99 \\
\hline Chocó & 12,7 & 10,64 & 0,86 & 0,4 & 32.355 .349 & 67,33 & 81,51 & 48,05 \\
\hline Guainía & 64,9 & 0,57 & 0,79 & 0,0 & 20.235 .072 & 17,14 & 75,91 & 35,50 \\
\hline Guajira & 44,9 & 44,62 & 0,80 & 1,0 & 27.153 .565 & 78,89 & 72,88 & 45,95 \\
\hline Guaviare & 4,3 & 2,05 & 0,75 & 0,1 & 27.007 .027 & 64,71 & 53,38 & 41,52 \\
\hline Putumayo & 20,9 & 13,70 & 0,76 & 0,4 & 31.625 .353 & 59,26 & 73,99 & 64,26 \\
\hline Quindío & 0,4 & 304,67 & 0,42 & 0,8 & 53.049 .227 & 92,53 & 72,40 & 75,16 \\
\hline San Andrés & 0,1 & 1457,71 & 0,38 & 0,2 & 142.795 .597 & 31,10 & 70,28 & 60,46 \\
\hline Sucre & 11 & 77,24 & 0,73 & 0,8 & 73.916 .838 & 73,35 & 112,06 & 74,19 \\
\hline Vaupés & 66,6 & 0,80 & 0,78 & 0,0 & 19.318.273 & 83,55 & 63,33 & 38,09 \\
\hline Vichada & 44,4 & 0,70 & 0,84 & 0,1 & 21.220 .299 & 42,35 & 80,09 & 34,58 \\
\hline Promedio nacional & 24,42 & 148,43 & 0,70 & 0,38 & 41.116 .321 & 61,70 & 75,72 & 53,01 \\
\hline
\end{tabular}

FIG. 2/ Departamentos de Colombia en los lugares más bajos de acuerdo con indicadores sociales y económicos.

habita en las cabeceras o en las zonas rurales, la densidad poblacional, las actividades económicas más importantes, el nivel de acceso a la educación superior, la cobertura de los servicios básicos, los usos del suelo y los tiempos de viaje en automóvil por carretera principal hasta las cabeceras de los territorios urbanos.

En todos estos análisis se buscaba medir el nivel de ruralidad y los vínculos de los territorios rurales con los centros poblados de carácter urbano, con el fin de determinar las lógicas y vínculos territoriales existentes, más allá de la simple consideración de las divisiones administrativas definidas estatalmente. El propósito era comprender lo que sucede con los territorios mediante el establecimiento de redes de trabajo y el movimiento de las personas, y los efectos que estos pueden generar en los niveles de avance o estancamiento económico y social de cada territorio. A partir de la información de base, los documentos consultados clasificaron a los municipios y las áreas no municipalizadas en categorías que se distribuyen entre lo urbano y lo rural, y que derivaron en diferentes clases dependiendo de la combinación de estos dos elementos o la prevalencia de alguno de ellos sobre el otro (Ver FIG. 4).

En los tres casos, las áreas no municipalizadas fueron clasificadas en las categorías más rurales. En el caso de Ramírez (2016) quedaron incluidas como Provincias Rurales Alejadas y Aisladas, lo cual significa que se requieren más de cuatro horas de viaje por carretera para llegar hasta la cabecera de provincia urbana o intermedia más cercana. CARRIAZO \& REYES (2012) por su parte clasificaron las áreas no municipalizadas en la categoría de Profundamente Rurales o Rurales Dispersos, los cuales en general son territorios aislados, donde no hay conglomeración, por lo que no existen desplazamientos de personas desde o hacia el territorio. Asimismo, PosadA (2010) las clasificó como Eminentemente Rurales en donde se incluyen los territorios con población inferior a 5.804 habitantes, caracterizados por ser principalmente rurales y económicamente insuficientes. Estos territorios también se caracterizan porque su actividad económica está basada principalmente en el sector agropecuario con leve aprovechamiento de los recursos forestales y donde existe una alta cobertura de energía, media en acueducto y baja en alcantarillado.

\section{Normas recientes sobre territorios indígenas y áreas no municipalizadas}

Al margen de estas particularidades, los departamentos con áreas no municipalizadas se administran a partir de los marcos definidos para los demás departamentos en Colombia, y la gestión de los asuntos locales responde a la misma normatividad que rige para el nivel municipal. Por lo tanto, debido a que las autoridades que tradicionalmente han gobernado estas zonas geográficas lo han hecho a la luz de los preceptos definidos desde 


\begin{tabular}{|c|c|c|}
\hline Título & Criterios de clasificación & Categorías municipales \\
\hline $\begin{array}{l}\text { Configuración territo- } \\
\text { rial de las provincias } \\
\text { de Colombia (2016) } \\
\text { Juan Carlos Ramírez } \\
\text { y J. Johan Manuel } \\
\text { de Aguas P. }\end{array}$ & $\begin{array}{l}\text { - Densidad poblacional de la provincia } \\
\text { - Proporción de la población de la provincia en } \\
\text { sus cabeceras municipales } \\
\text { - Tamaño de la población en la cabecera mayor } \\
\text { - Tiempo de viaje por carretera hasta la cabe- } \\
\text { cera de provincia urbana }\end{array}$ & $\begin{array}{l}\text { - Provincias urbanas; } \\
\text { - Provincias Intermedias que se clasifican en i) in- } \\
\text { termedias periurbanas, ii) intermedias cercanas, } \\
\text { e iii) intermedias alejadas } \\
\text { - Provincias rurales, las cuales a su vez se subdi- } \\
\text { viden en i) rurales periurbanas, ii) rurales cercanas } \\
\text { a urbanas, iii) rurales cercanas a intermedias, y } \\
\text { iv) rurales alejadas y aisladas. }\end{array}$ \\
\hline
\end{tabular}

Territorios funcionales: · Porcentaje de la población que vive en el resto • Regiones metropolitanas o territorios totalmenun análisis del gradien- rural, te urbanos.

te rural-urbano - Densidad de población, - Territorios rurales-urbanos con núcleo principalpara Colombia, (2012) - Número de entidades financieras diferentes mente urbano

Fernando Carriazo al banco agrario, - Territorios rurales-urbanos con núcleo parcial-

y Mónica Juliana Reyes · Actividad económica donde se describen ran- mente rural

gos del total de la población ocupada en ac- - Territorios ruralesurbanos con núcleo (parcial, tividad primaria principal o totalmente urbanos)

- Porcentaje de la población que accede a edu- - Territorios profundamente rurales plurimunicipacación superior les

- Territorios profundamente rurales o rurales dispersos.

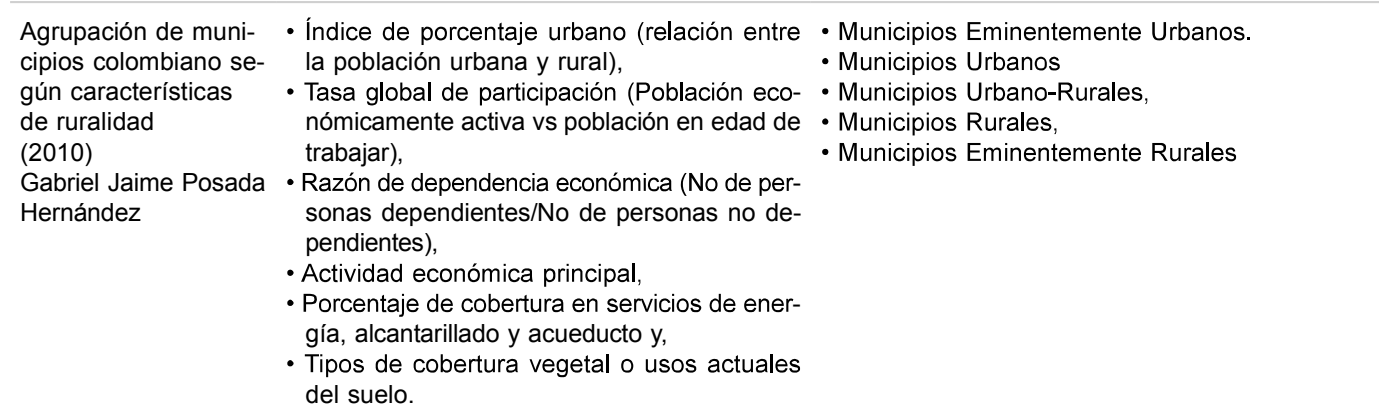

FIG. 3/ Estudios académicos de clasificación de los municipios y las áreas no municipalizadas en Colombia.

Fuente: Elaboración propia, con base en los documentos relacionados.

el centro y no a partir de las ópticas culturales, y económicas propias, el manejo de los asuntos locales ha demostrado ser bastante defectuoso. Esto se ha debido, tanto a la distancia existente entre la población y la administración del nivel intermedio, como a las dificultades de las burocracias departamentales para interpretar los asuntos propios de las comunidades (HURTADO, 2010).

Dadas estas circunstancias, el gobierno nacional expidió recientemente algunos decretos cuya naturaleza podría derivar en beneficios tanto de las áreas no municipalizadas, en tanto unidades territoriales, como de las comunidades indígenas que se encuentran en su interior y al interior de otros territorios ya formalizados. Este es el caso de los Decretos 1953 de 2014, 632 de 2018 y 1454 de 2018. Los dos primeros, se crearon para definir un régimen especial para los denominados Territorios Indígenas, en ausencia de la aprobación de las ETIs de que debió ocuparse la LOOT y para desarrollar el artículo transitorio $\mathrm{N}^{\circ} 56$ de la $\mathrm{CP}$ de
1991. Este artículo autoriza al gobierno nacional para dictar normas fiscales y otras relativas al funcionamiento de los territorios indígenas y su coordinación con las demás entidades territoriales.

En este marco, los Decretos 1953/14 y 632/2018 reconocen a los Territorios Indígenas como una organización político-administrativa de carácter especial a la que se le asignan unas funciones para ser ejercidas por sus autoridades propias. Estas normas avanzan en que, además de los recursos de la asignación especial del Sistema General de Participaciones ${ }^{3}$, orientados a financiar los proyectos de inversión establecidos en los planes de vida de los resguardos indígenas, estos territorios tienen la posibilidad de administrar directamente los recursos orientados a salud, educación y agua potable que en el pasado tenían que pasar por la administración de los departamentos o de los municipios certificados a los que pertenecían. Se trata de normas que buscan suplir la ausencia de las ETIs mientras son 
autorizadas a través de la LOOT y prácticamente permiten que, aunque no estén formalizados desde la Constitución, los Territorios Indígenas funcionen con todas las características que tendrían como entidad territorial, autoridades, funciones y recursos.

El Decreto 1953/14 avanzó en la definición de los contenidos más importantes de esta materia dirigida para los Territorios Indígenas que se organizaran en cualquier parte del territorio nacional, pero el 632/18 se creó con el propósito de reglamentar exclusivamente los Territorios Indígenas en las áreas no municipalizadas de los departamentos de Amazonas, Guainía y Vaupés, tal como lo establece su artículo $1^{\circ}$ :

"El presente Decreto establece las normas transitorias relativas a la puesta en funcionamiento de los territorios indígenas ubicados al interior de los resguardos en áreas no municipalizadas de los departamentos de Amazonas, Guainía y Vaupés; los mecanismos para el relacionamiento entre los gobiernos propios de los pueblos indígenas ubicados en estos territorios y las disposiciones para su organización fiscal y su coordinación con las entidades territoriales, en tanto se expide la ley de que trata el artículo 329 de la Constitución Política. En todo caso reconociendo, que el beneficiario de la Asignación Especial del Sistema General de Participaciones es el resguardo indígena de conformidad con la establecido en el artículo 356 de la Constitución Política."

El último de estos decretos (1454 de 2018), sin embargo, tiene una orientación distinta, ya que emite un concepto desde el gobierno nacional en el que aprueba la conversión de cinco de las áreas no municipalizadas (Barrancominas y Mapiripana; y Pana Pana, San Felipe y Puerto Colombia) del departamento de Guainía en dos nuevos municipios, Mapiripana y San Felipe. Como veíamos antes (FIG. 1) este departamento es el único del territorio nacional que hasta el momento ha contado con un solo municipio. La creación de estos dos municipios no incorpora el reconocimiento de autonomías indígenas en tanto su manejo se haría dentro del marco normativo que rige para los municipios en general. Lo anterior, no obstante, no excluye la posibilidad de que a su interior se reconozcan o se conserven Territorios Indígenas en las condiciones de las normas que los regulan.

Aunque la evolución de que han sido objeto recientemente las áreas no municipalizadas muestra la marcha lenta de nuestro Estado, en cabeza del ejecutivo, hacia su reconocimiento y formalización,

${ }^{3}$ El Sistema General de Participaciones (SGP) es el sistema de transferencias intergubernamentales definidos para Colombia en el que la nación transfiere recursos a las entidades también pone en evidencia las resistencias que todavía están presentes en el legislativo puesto que dicha formalización involucra principalmente el reconocimiento de los derechos de las comunidades indígenas que los habitan (BAENA, 2015). En especial debido a que la alternativa más apro-piada que daría lugar a la legalización territorial de esta realidad social latente, que sería la reforma de la LOOT para incluir las ETIs es poco probable, más tomando en cuenta que su expedición se logró después de que fueran presentados diecinueve proyectos de ley.

\section{El reconocimiento de los territorios indígenas a nivel latinoamericano}

Los avances en el reconocimiento de los derechos en materia territorial para los pueblos indígenas que se han realizado en Colombia, aunque limitados, son similares a los que se han llevado a cabo por otros países latinoamericanos. Los mismos se enmarcan en normas internacionales como el Convenio N ${ }^{\circ} 169$ de la OIT (1989) y en la Declaración de las Naciones Unidas sobre los Derechos de los Pueblos Indígenas (2007). Dichas normas se produjeron como resultado de los movimientos étnicos, por medio de los cuales estos pueblos han buscado la generación de espacios de participación en las decisiones públicas en pro del reconocimiento de sus derechos. Como resultado de los primeros logros, varios estados incluyeron en sus constituciones, en los años 80 y 90 , el reconocimiento de los pueblos indígenas como sujetos colectivos de derechos políticos que deberían ser expresados en espacios de participación, el ejercicio de su autonomía y autodeterminación y el derecho al territorio (AyLWIN, 2014) (Ver FIG. 4).

En lo que respecta al derecho de los indígenas al territorio, éste fue considerado desde las normas internacionales a partir de elementos como la tierra, los territorios y los recursos naturales allí ubicados. Estos tres elementos son tomados en cuenta de manera articulada en tanto representan los vínculos de carácter cultural y espiritual que une a los pueblos indígenas con su entorno (CEPAL, 2014). Además, esta consideración debe partir de que la tierra no puede ser entendida apenas como un objeto sobre el cual se ejerce posesión y usufructo, sino que se trata de un elemento de carácter material del cual depende la preservación de la herencia cultural que caracteriza a estos pueblos 


\begin{tabular}{ll}
\multicolumn{1}{c}{ Derechos reconocidos } & \multicolumn{1}{c}{ Estados } \\
\hline $\begin{array}{l}\text { Reconocimiento constitucional de la naturaleza pluricultural, } \\
\text { multicultural o multiétnica de los estados }\end{array}$ & $\begin{array}{l}\text { Colombia, 1991; México 1992 y 2001; Perú, 1993; Bolivia, } \\
1994 \text { y 2009; Ecuador, } 1998 \text { y 2008 }\end{array}$ \\
\hline $\begin{array}{l}\text { Derecho consuetudinario, tanto dentro de la jurisdicción del } \\
\text { Estado como a través de los sistemas de justicia indígena }\end{array}$ & México, Ecuador, Colombia y Perú \\
\hline $\begin{array}{l}\text { Derechos de representación política especial indígena } \\
\begin{array}{l}\text { Derechos de autonomía o autogobierno de los pueblos indí- } \\
\text { genas en figuras como resguardos, entidades territoriales } \\
\text { indígenas, circunscripciones territoriales indígenas, municipios }\end{array}\end{array}$ & $\begin{array}{l}\text { Colombia, Ecuador, México, como habían sido establecidos } \\
\text { poragua en 1987 }\end{array}$ \\
\hline
\end{tabular}

FIG. 4/ Derechos que han sido reconocidos constitucionalmente a los pueblos indígenas en países latinoamericanos.

Fuente: Elaborado con base en AyLWIN (2014)

y la posibilidad de transmitirla para sus generaciones futuras (CID, 2009).

Para garantizar estos propósitos, estas normas establecieron que los estados debían otorgar, a los pueblos interesados, el derecho de posesión sobre las tierras que han ocupado tradicionalmente. Incluso aunque se trate de tierras que no han sido ocupadas por ellos, los estados deberían garantizar la utilización de territorios sobre los que han tenido acceso en el pasado y que son importantes para sus actividades tradicionales y de subsistencia. Asimismo, se plantea que también se debe proteger el derecho a utilizar, administrar y conservar los recursos naturales presentes en su territorio y en los casos en que el Estado sea el propietario del suelo y del subsuelo, de todas maneras se debería asegurar la participación de los pueblos en los beneficios que se deriven de la explotación y aprovechamiento de los mismos y a recibir una indemnización por los daños que las comunidades indígenas puedan sufrir como resultados de estas actividades.

Pero, a pesar de estas directrices internacionales y de los logros que se han reflejado en los diseños constitucionales de varios estados, los mismos se dieron en el contexto de la transformación neoliberal del sector público, por lo que en la práctica se generó lo que STAVENHAGEN (2005) denominó la brecha de la implementación. Esta brecha se refleja en la distancia existente entre lo establecido por las constituciones y los desarrollos normativos que se han derivado de la misma, en la falta de dispositivos suficientes que garanticen la exigibilidad de estos derechos reconocidos por la constitución, y finalmente por la ausencia de recursos y de interés político para adelantar políticas que los lleven a la práctica.

Aunque varios estados han reconocido el derecho sobre la tierra de los pueblos indígenas
(Colombia, Brasil, Ecuador, Bolivia, Colombia, Chile), todavía prevalecen defectos como la propiedad de los recursos que allí se generan, la consulta para que se permita a los estados la explotación de estos recursos o la participación en los beneficios derivados de dicha explotación (AYLWIN, 2014). Estas dificultades han hecho además que el reconocimiento de autonomías para la gestión de los asuntos propios de estos pueblos, mediante la creación de entidades formales dentro de los marcos territoriales de los estados, sea todavía más limitado.

En algunos de estos casos el reconocimiento llega solo al nivel de la Constitución como en Colombia, que como veíamos antes plantea la posibilidad de crear ETIs, pero a la fecha no las ha concretado. Algo similar sucedió con la Constitución de 1998 en Ecuador donde se incluyeron las Circunscripciones Territoriales Indígenas (TCO), las cuales nunca fueron reglamentadas legalmente (RodRíguez, 2014). No obstante, existen otros casos donde se han logrado mayores avances como en Bolivia, reconocido como un estado plurinacional, donde se crearon las Autonomías Indígenas Originario Campesinas (AIOC) que se pueden reconocer por la vía municipal como sucedió en 2010 , o a través de los Territorios Indígenas Originario Campesinos (TIOC) los cuales, sin embargo, requieren un procedimiento especial que no ha sido reglamentado hasta la fecha (RodRíguez, 2014).

No obstante, los casos más avanzados en esta materia son Panamá y Nicaragua. El primero de ellos configuró las denominadas comarcas indígenas cuya existencia data del año 1953. Estas entidades, que en la actualidad son 5 , tienen la facultad de expedir sus propias leyes constitutivas a través de las cuales se definen su estructura política administrativa tradicional, sus autoridades, sus competencias, el manejo de los recursos naturales 
y las particularidades que definen su identidad y sus valores culturales (MoLINA \& FIGUEROA, \& QUISBERT, 2005). Nicaragua por su parte estableció las regiones autónomas, las cuales se encuentran definidas desde la CP de 1987, y reglamentadas mediante la Ley 28 del mismo año. Estas normas les reconocen a estas entidades y a las comunidades que se asientan en su interior el derecho a sus propias formas organizativas en materia social, económica y cultural y a la libertad para elegir a sus autoridades.

Las regiones autónomas fueron reglamentadas como entidades descentralizadas desde el año 2003, cuando se les hizo partícipes de los programas de salud, justicia, transporte y educación, entre otros. Sin embargo, la autonomía reconocida por la Constitución a estas regiones no es solamente una autonomía indígena, sino una autonomía de base multiétnica que buscó garantizar la participación e incidencia en el gobierno regional por parte de los diferentes grupos étnicos que habitan el territorio, y que incluyen indígenas, afrodescendientes y mestizos (AYLWIN, 2014).

\section{Conclusiones}

Los corregimientos departamentales o áreas no municipalizadas en Colombia son una realidad que se encuentra en el territorio pero que no está representada en nuestro diseño normativo, por lo que actualmente constituyen un limbo jurídico. Los diferentes intentos por formalizarlos, bien sea mediante su incorporación a los municipios existentes, o su legalización como municipios o como ETIs no han sido exitosos, debido a las particularidades económicas, sociales y geográficas que los identifican y que han dificultado su consideración dentro del conjunto de entidades de nivel local de nuestro país. Estas particularidades ubican a estos territorios en los peores lugares o muy por debajo si se les compara con las demás áreas correspondientes al nivel local, o incluso si se les analiza como particularidades de los departamentos de los que forman parte.

Debido a la ausencia de formalización y a la urgente necesidad de reconocerlas el ejecutivo nacional ha optado por avanzar en la entrega, a los Territorios Indígenas allí constituidos, de cada vez mayores derechos que son propios de las entidades territoriales en materia de autoridades propias, competencias propias y recursos por transferencias. Estos avances, que se articulan con las directrices iniciales definidas desde la Constitución Política de 1991, han procurado progresar en el otorgamiento de autonomías principalmente a partir del manejo de los recursos provenientes del nivel nacional. Esto convierte a los Territorios Indígenas inicialmente en unidades de tipo administrativo, más que entes autónomos reflejo de las comunidades que los habitan. Si bien esta es la manifestación del interés del Estado en cabeza del ejecutivo por contribuir al reconocimiento de estos pueblos también evidencia el desinterés del legislativo en dicho proceso.

Este fenómeno, sin embargo, no solo es característico de nuestro país, sino que se puede ver a lo largo de la región latinoamericana donde habitan pueblos indígenas. A pesar de la existencia de directrices internacionales que en las últimas tres décadas han apuntado a la reivindicación de los derechos de estos pueblos, solo algunos países han realizado reformas constitucionales para su reconocimiento. Pero son todavía menos aquellos que han avanzado en el reconocimiento de sistemas de justicia propios, en el otorgamiento de tierras y reconocimiento de los beneficios económicos derivados de ellas, y muy pocos son los que han logrado la creación de entidades territoriales autónomas.

Aunque Colombia ha realizado avances importantes en todos estos temas, todavía existen resistencias para la formalización de los territorios donde habitan muchas de las comunidades indígenas de nuestro país, debido, entre otras razones, a que los mismos no han alcanzado históricamente las condiciones propias de los demás territorios de nivel local. Con ello se pone en evidencia la ausencia de un marco normativo totalmente acabado que refleje las particularidades propias de estos territorios, y que mejore las posibilidades para la gestión y la potenciación del bienestar económico y social de sus habitantes.

\section{Bibliografía}

AyLWIN, J. (2014): Los derechos de los pueblos indígenas en América Latina: avances jurídicos y brechas de implementación. Red de Derechos Humanos y Educación Superior, Barcelona, 275-300.

Baena Carrillo, S. (2015): La autonomía de las Entidades Territoriales Indígenas. Revista digital de Derecho Administrativo, n. ${ }^{\circ} 13$, primer semestre/2015, pp. 99-133.

BARABÁs, A. (2003): Diálogos con el territorio: simbolizaciones sobre el espacio en las culturas indígenas de México, 4 Vol. México: INAH.

Carmona, C \& Supelano, D. \& Osejo, I (2014): Tipologías Departamentales y Municipales: una propuesta para comprender las entidades territoriales colombianas, Grupo de Estudios Territoriales.

CARRIAZO, F., \& ReYes, M. (2012): Territorios funcionales: un análisis del gradiente rural-urbano para Colombia (No. 146467). Universidad de Los Andes, 
Economics Department.

DEMARCHI, F. (1983): II territorio come fornitore di referenti simbolici. FAE riviste.

FLORES, M. (2007): La identidad cultural del territorio como base de una estrategia de desarrollo sostenible. Ópera, 7 (7), 35-54.

GimÉNEZ, G. (2005): Territorio e identidad. Breve introducción a la geografía cultural. Trayectorias, 7(17).

GonZÁlEZ, L. (2010): Conocimiento y control en los confines del territorio nacional: hacia la construcción de un saber territorial, 1850-1950. Historia y Sociedad (Colombia), (19), 123-143.

GuARDERAs, A. B. (2007): Estado, sociedad y territorio: el debate actual sobre descentralización y autonomías en la región andina. Nueva sociedad, 210, 189-202.

HeRnándeZ, A. (2015): Objetivos Inéditos de la categorización municipal. En Varios autores (2015). Problemática de los municipios pequeños en Colombia ¿Supresión o reforma? Universidad Externado de Colombia.

- (2018): Uniformidad y diversificación de los gobiernos municipales en Colombia. Revista de la Facultad de Derecho de México, 68(271), 151-168.

Hernández, P. (1999): Descentralización, desconcentración y delegación en Colombia. Legis Editores.

HuRTADO, L. (2010): Ordenamiento territorial en corregimientos departamentales. Los casos de MiritiParaná, Tarapacá y El municipio de Puerto Nariño en el departamento del Amazonas. En CoLmenARES et al. (2010). Ecología política de la Amazonia. Las profusas y difusas redes de la gobernanza, 435-465. Universidad Nacional de Colombia, Sede Amazonía.

Molina, R.\& FigueROA, M., \& QUISBERT, I. (2005): Pueblos indígenas de Bolivia: diagnóstico sociodemográfico a partir del censo del 2001. In CEPAL Documentos de proyectos (No. 24). ONU/CEPAL.

NACIONES UnIDAS (2007): Declaración de las Naciones Unidas sobre los derechos de los pueblos indígenas.

Organización InTERnacional del Trabajo (OIT) (1989): Convenio Núm. 169 de la OIT sobre Pueblos Indígenas y Tribales.

Pérez, J. F. P. (2010): Génesis del municipio. Multidisciplina, (7).

Posada, G. J. (2010): Agrupación de municipios colombianos según características de ruralidad. Universidad Nacional de Colombia, Medellín.

Raffestin, C. (1980): Pour une géographie du pouvoir. ENS Editions.

Ramírez, J. C. \& de Aguas, J. M. (2016): Configuración territorial de las provincias de Colombia. Santiago, Chile. CEPAL.

Rodríguez, E. C. (2014): Estado plurinacional, interculturalidad y autonomía indígena: Una reflexión sobre los casos de Bolivia y Ecuador. Revista Via luris, (14), 55-71.

SIXIERI, C. (2014): Federalismo y centralismo en los orígenes de la Colombia contemporánea. História (São Paulo), 33 (2)

Stavenhagen, R. (2005): Report of the Special Rapporteur on the situation of human rights and fundamental freedoms of indigenous people. Australian Indigenous Law Reporter, 9 (1), 102-113.

UNICEF (2003): Los pueblos indígenas en Colombia: Derechos, políticas y desafíos. UNICEF.

\section{Referencias normativas}

Acto legIsLATIVo 01 dE 1936: PUBLICADO EN EL DIARIO OFICIAL No. 23.263 DE 22 dE AGOSTO DE 1936.

Acto Legislativo 01 de 1986: Publicado en el Diario Oficial No. 37.304 del 10 de enero de 1986

Асто Legislativo 01 De 1945: Publicado en el Diario Oficial, Bogotá 17 de febrero de 1945.

Comisión Económica para AmÉrica Latina y EL CARIBE (CEPAL) (2014): Los pueblos indígenas en América Latina, Avances en el último decenio y retos pendientes para la garantía de sus derechos. Naciones Unidas, CEPAL.

Constitución de la República de la Nueva Granada (1843): 8 de mayo de 1843. República de la Nueva Granada.

- (1843): Congreso de la República, 20 de mayo de 1853. República la Nueva Granada.

Constitución DE los Estados UnIDOS dE Colombia (1863): Cámara de Representantes, Colombia 8 de mayo de 1863.República de los Estados Unidos de Colombia.

Constitución Politica de Colombia (1886): Asamblea Nacional Constituyente, Bogotá. República de Colombia (1886)

- (1991): Asamblea Nacional Constituyente, Bogotá Colombia 6 de julio de 1991. República de Colombia.

Constitución Política del Ecuador (1998): Asamblea Nacional Constituyente. Decreto Legislativo No. 000. RO/ 1 de 11 de agosto de 1998. República del Ecuador.

Constitución Política del Estado de Bolivia (2009): República de Bolivia (2009).

Constitución Política de Nicaragua (1987): Aprobada el 19 de noviembre de 1986. República de Nicaragua (1987).

Contaduría General de la Nación (2018): Resolución 556 del 20 de noviembre de 2018, "Por la cual se expide la certificación de categorización de las entidades territoriales (Departamentos, Distritos y Municipios conforme a lo dispuesto en las Leyes 136 de 1994 , 617 de 2000 y 1551 de $2012 "$

DANE, 2017: Cuentas Departamentales -CD Producto Interno Bruto, 2016. Boletín Técnico. Bogotá, D.C.

DECRETo 2451: Por el cual se reglamentan las disposiciones de la Ley $2^{a}$ de 1943 , sobre administración de las Intendencias y Comisarias. Diario Oficial N. 25431. Presidencia de la República (1943).

DECRETO 2274: Por el cual se dictan normas tendientes a asegurar la debida organización y funcionamiento de las entidades territoriales erigidas como departamentos en la Constitución Política y se dictan otras disposiciones. Diario Oficial No 40.078. Presidencia de la República (1991).

DECRETO 1421: Por el cual se dicta el régimen especial para el Distrito Capital de Santafé de Bogotá. Presidencia de la República (1993).

DECRETO 1953: Por el cual se crea un régimen especial con el fin de poner en funcionamiento los Territorios Indígenas respecto de la administración de los sistemas propios de los pueblos indígenas hasta que el Congreso expida la ley de qué trata el artículo 329 de la Constitución Política. Diario Oficial No. 49297 del 07 de octubre de 2014. Presidencia de la República (2014). 
DeCRETo 632: Por el cual se dictan las normas fiscales y demás necesarias para poner en funcionamiento los territorios indígenas ubicados en áreas no municipalizadas de los departamentos de Amazonas, Guainía y Vaupés. Presidencia de la República (2018).

DeCRETo 1454: Por el cual se emite visto bueno para la creación de los municipios de Barrancominas y San Felipe en el departamento de Guainía. Presidencia de la República (2018).

Departamento Nacional de Planeación -DNP (2017): "Desempeño fiscal de los departamentos y municipios 2016" Bogotá, Colombia.

- (2018): "Desempeño fiscal de los departamentos y municipios 2017" Bogotá, Colombia.

LEY 2 DE 1943: Por la cual se dictan algunas disposiciones sobre administración, división administrativa y régimen electoral de las Intendencias y Comisarías. Diario Oficial N 25342.

LEY 1454 DE 2011: Por la cual se dictan normas orgánicas sobre ordenamiento territorial y se modifican otras disposiciones. Diario Oficial No. 48.115 de 29 de junio de 2011.

LEY 1551 dE 2012: Por la cual se dictan normas para modernizar la organización y el funcionamiento de los municipios. Diario Oficial No. 48.483 de 6 de julio de 2012.

Sentencia C-141-01. Corte Constitucional de Colombia (2001).

SENTENCIA C-100-2013: Normas tendientes a modernizar la organización y el funcionamiento de los municipios-extensión a los departamentos de la facultad para celebrar convenios con las juntas de acción comunal. Corte Constitucional de Colombia (2013). 